\title{
ESTRUTURA PIEZELÉTRICA MULTIFREQUENCIA PARA APLICAÇÃO EM VIBRAÇÕES DE BANDA ESTREITA
}

\author{
FERNANDO H. O. CAMARA ${ }^{1}$, JOÃO A. PEREIRA², ANTONIO E. TURRA ${ }^{2}$.
}

\author{
1. Departamento de Engenharia Mecânica, Universidade Tecnológica Federal do Paraná \\ Avenida Alberto Carazzai, 1640, 86300-000, Cornélio Procópio, Paraná \\ E-mails: fernandocamara@utfpr.edu.br
}

\author{
2. Grupo de Materiais e Sistemas Inteligentes, Depto. de Engenharia Mecânica, Universidade Estadual \\ Paulista \\ Avenida Brasil, 56,15385-000, Ilha Solteira, São Paulo \\ E-mails: japereira@dem.feis.unesp.br,turra@dem.feis.unesp.br
}

\begin{abstract}
The use of piezoelectric materials in converting mechanical energy from the vibrations into electrical energy has increased considerably in recent years mainly due to high energy conversion capacity of these materials. However, most of the works shown in the literature exhibit piezoelectric structures and devices for energy harvesting that meets the requirements generation and energy storage only when operating at resonance, when operating in conditions of excitation slightly different from resonance the electrical energy converted decreases dramatically. This paper presents the configuration of a piezoelectric structure called multifrequency piezostructure that enables the generation of energy in a reasonable amount even when there are variations in the operating conditions of the structure. The multifrequency piezostructure behavior is obtained by simulations in ANSYS and the frequency band of operation is defined using the concept of half-power bandwidth. The numerical results of the model are compared with experimental data showing its potential application.
\end{abstract}

Keywords_Multifrequency Piezostructure, Energy Harvesting, Mechanical Vibrations.

Resumo- O uso de materiais piezelétricos na conversão de energia mecânica, proveniente das vibrações, em energia elétrica tem aumentado consideravelmente nos últimos anos principalmente devido a alta capacidade de conversão de energia desses materiais. Entretanto, grande parte dos trabalhos mostrados na literatura apresentam dispositivos e estruturas piezelétricas de energy harvesting que atende os requisitos de geração e armazenamento de energia apenas quando operam na ressonância, quando operam em condições de excitação ligeiramente diferentes da ressonância a energia elétrica convertida diminui drasticamente. O presente trabalho apresenta a configuração de uma estrutura piezelétrica denominada piezoestrutura multifrequência que possibilita a geração de energia em uma quantidade razoável mesmo quando ocorrem variações nas condições de operação da estrutura. O comportamento da piezoestrutura multifrequência é obtido através de simulações no software ANSYS e a banda de frequência de operação é definida utilizando o conceito de banda de meia potência. Os resultados numéricos do modelo são comparados com dados experimentais mostrando sua potencialidade de aplicação.

Palavras-chave— Piezoestrutura multifrequência, Energy harvesting, Vibrações mecânicas.

\section{Introdução}

A utilização de materiais piezelétricos para conversão de energia mecânica proveniente das condições de vibrações em energia elétrica tem aumentado drasticamente na última década, buscando atender a demanda por fontes alternativas de energia, principalmente para a alimentação de sistemas de monitoramento da condição estrutural (SHM) bem como dispositivos de aeronaves não tripuladas (UAV's), tornando-os dispositivos autônomos. Grandes esforços tem sido e continuam sendo empregados no desenvolvimento e estudo de técnicas de extração e armazenamento de energia buscando aumentar o potencial elétrico gerado pelas estruturas piezelétricas (Adachi e Tanaka, 2009; Ajitsaria et al., 2007), bem como minimizar o potencial elétrico perdido pela conversão AC/DC (Lefreuve, 2006; Ramadass, 2010; Souza, 2011). Aliado a isso, existe ainda um grande interesse no projeto de sistemas de sensores de baixo consumo (Ferrari et al., 2009; Johnson,
2006) o que torna o desenvolvimento de sistemas autônomos praticamente uma realidade atual.

Um sistema autônomo de extração e armazenamento de energia utilizando elemento piezelétrico pode ser basicamente dividido em quatro partes: o gerador piezelétrico de energia, o circuito extrator/coletor, um dispositivo de armazenamento e o sistema alimentado pela energia disponível no dispositivo de armazenamento. Nos diversos trabalhos desenvolvidos até o momento o gerador piezelétrico produz alto potencial elétrico quando operado na frequência de ressonância. Porém, o nível do potencial elétrico diminui drasticamente à medida que se afasta da frequência de ressonância. O presente trabalho foca o desenvolvimento de um gerador piezelétrico multifrequência que busca manter o nível de energia elétrica gerada mesmo que ocorra variações das condições de operação e a correspondente oscilação da frequência. 


\section{Modelagem da Estrutura Piezelétrica}

\subsection{Efeito Piezelétrico}

$\mathrm{O}$ interesse na aplicação de materiais piezelétricos para conversão de energia mecânica em energia elétrica teve um aumento drástico nos últimos anos não só devido à necessidade de fontes alternativas de alimentação para sistemas de SHM e dispositivos de UAV's, mas também pela alta capacidade dos materiais piezelétricos em converter a energia mecânica em energia elétrica (DuTOIT, 2007). O efeito piezelétrico pode ser descrito através das equações constitutivas (DuTOIT, 2007) abaixo, neste caso para o modo de operação 31 .

$$
\begin{aligned}
& D_{3}=d_{31} T_{1}+\varepsilon_{33} E_{3} \\
& S_{1}=s_{11} T_{1}-d_{31} E_{3}
\end{aligned}
$$

Onde, $D$ é o deslocamento elétrico, $T$ a tensão mecânica, $\varepsilon$ a permissividade elétrica, $s$ a compliância elástica, $S$ a deformação mecânica, $d$ o coeficiente piezelétrico e $E$ o campo elétrico.

\subsection{Modelo de elementos finitos}

Em diversos modelos de parâmetros concentrados apresentados na literatura é possível predizer a energia elétrica gerada com precisão. Entretanto, alguns problemas como, por exemplo, modelagem simplificada e problemas na formulação do acoplamento piezelétrico (Erturk e Inman, 2008b) são frequentemente encontrados nesses modelos até então apresentados. Buscando apresentar um modelo numérico mais fiel ao modelo experimental, o presente trabalho utiliza-se do método de elementos finitos através do software comercial ANSYS.

As equações acopladas governantes do movimento são obtidas a partir do princípio da mínima energia por meio do princípio variacional e podem ser representadas na forma matricial:

$$
\begin{gathered}
{\left[\boldsymbol{M}_{\boldsymbol{u u}}\right]\{\ddot{\boldsymbol{u}}\}+\left[\boldsymbol{C}_{u u}\right]\{\boldsymbol{u}\}+\left[\boldsymbol{K}_{u u}\right]\{\boldsymbol{u}\}+\left[\boldsymbol{K}_{u \varphi}\right]\{\boldsymbol{\varphi}\}=\{\boldsymbol{F}\}} \\
-\left[\boldsymbol{C}_{\boldsymbol{\varphi} \varphi}\right]\{\dot{\boldsymbol{\varphi}}\}+\left[\boldsymbol{K}_{u \varphi}\right]^{t}\{\boldsymbol{u}\}-\left[\boldsymbol{K}_{\boldsymbol{\varphi} \varphi}\right]\{\boldsymbol{\varphi}\}=\{\boldsymbol{Q}\}
\end{gathered}
$$

Sendo, $\boldsymbol{u}$ o deslocamento mecânico que descreve o movimento em cada elemento estrutural e $\varphi$ os graus de liberdade elétricos que descrevem o potencial elétrico nos nós estruturais. Com $\left[\boldsymbol{M}_{u u}\right]$ sendo a matriz elementar de massa, $\left[\boldsymbol{K}_{u u}\right]$ a matriz elementar de rigidez. $\left[\boldsymbol{K}_{\varphi \varphi}\right]$ é a matriz elementar dos coeficientes de permissividade dielétrica, $\left[\boldsymbol{C}_{\varphi \varphi}\right]$ é a matriz elementar do amortecimento dielétrico (capacitância), $\{\boldsymbol{Q}\}$ é o vetor das cargas nodais, da superfície e do corpo, $\left[\boldsymbol{K}_{u \varphi}\right]$ é a matriz do acoplamento piezelétrico, $\{\boldsymbol{F}\}$ é o vetor das forças nodais e de superfície que são aplicadas no sistema. A matriz de amortecimento $\left[\boldsymbol{C}_{\boldsymbol{u} u}\right]$, neste caso foi considerada como sendo proporcional às matrizes de massa e rigidez,

$$
\left[\boldsymbol{C}_{\boldsymbol{u u}}\right]=\alpha\left[\boldsymbol{M}_{\boldsymbol{u u}}\right]+\beta\left[\boldsymbol{K}_{\boldsymbol{u u}}\right]
$$

onde $\alpha$ e $\beta$ são coeficientes de proporcionalidade variando da ordem de $10^{-2}$ e $10^{-4}$, respectivamente.

\subsection{Piezoestrutura Multifrequência}

No caso de estruturas piezelétricas do tipo viga o fator de qualidade mecânica do elemento piezelétrico é comumente alto (Kok et al., 2011) e pequenas variações na frequência de excitação podem causar grandes perdas na energia elétrica gerada, o que representa uma grande limitação de uso desses dispositivos, principalmente, nos casos em que as condições de operação e geração de energia podem oscilar. Neste caso é proposto o desenvolvimento de uma estrutura piezelétrica multifrequência capaz de manter a mesma quantidade de energia gerada mesmo que a frequência de excitação oscile. Um arranjo composto por três conversores piezelétricos cobrindo uma faixa de frequências capaz de absorver diferentes mudanças e condições de operação é proposto e analisado. Inicialmente foi desenvolvido um primeiro conversor piezelétrico para operar em torno da frequência de ressonância do seu primeiro modo. $\mathrm{O}$ conversor piezelétrico consiste de uma estrutura do tipo viga excitada pela base com o elemento piezelétrico (PZT) colado próximo à base. A Figura 1 mostra esquematicamente a piezoestrutura analisada.

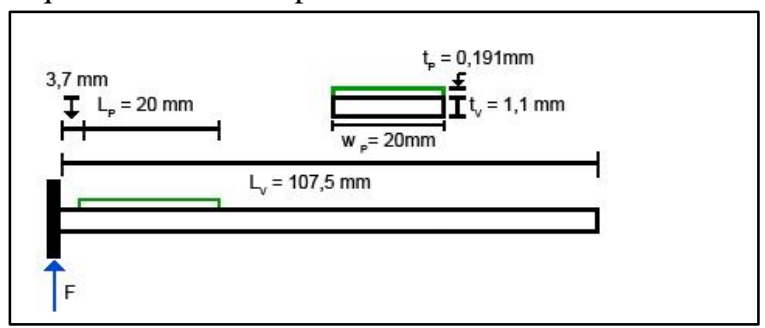

Figura 1. Conversor piezelétrico modelado por elementos finitos.

A estrutura foi avaliada numa condição de operação próxima a frequência do primeiro modo de vibração que é a condição de maior geração de energia deste tipo de estrutura.

$\mathrm{O}$ material escolhido para a viga hospedeira foi $\mathrm{o}$ acrílico devido ao seu baixo valor do módulo de Young que possibilita uma viga bastante flexível com uma deformação capaz de gerar um alto nível de energia e ocupe um espaço reduzido. As propriedades físicas e piezelétricas da estrutura e suas características geométricas são apresentadas na Tabela 1.

Tabela 1. Dimensões e propriedades do conversor piezelétrico.

\begin{tabular}{|l|c|l|c|}
\hline $\begin{array}{l}\text { Propriedades da } \\
\text { Viga }\end{array}$ & Valor & Propriedades do PZT & Valor \\
\hline Comprimento (mm) & 107.5 & Comprimento (mm) & 20 \\
\hline Espessura (mm) & 1.1 & Largura (mm) & 20 \\
\hline Largura (mm) & 20 & Espessura (mm) & 0.191 \\
\hline $\begin{array}{l}\text { Módulo de Young } \\
(\mathrm{GPa})\end{array}$ & 2.9 & $\begin{array}{l}\text { Módulo de Young } \mathrm{Y}_{1}^{\mathrm{E}} \\
(\mathrm{GPa})\end{array}$ & 62 \\
\hline $\begin{array}{l}\text { Densidade } \\
\left(\mathrm{kg} / \mathrm{mm}^{3}\right)\end{array}$ & 1150 & $\begin{array}{l}\text { Módulo de Young } \mathrm{Y}_{3}^{\mathrm{E}} \\
(\mathrm{GPa})\end{array}$ & 50 \\
\hline
\end{tabular}




\begin{tabular}{|l|l|l|c|}
\hline & & Constante piezelétrica & $650 \mathrm{e}-$ \\
& $\mathrm{d}_{33}(\mathrm{~m} / \mathrm{V})$ & 12 \\
\hline & Constante piezelétrica & $-320 \mathrm{e}-$ \\
$\mathrm{d}_{31}(\mathrm{~m} / \mathrm{V})$ & 12 \\
\hline & $\begin{array}{l}\text { Constante dielétrica } \\
\text { relativa } \mathrm{K}_{3}^{\mathrm{T}}\end{array}$ & 3800 \\
\hline & $\begin{array}{l}\text { Coeficiente Acopla- } \\
\text { mento } \mathrm{k}_{31}\end{array}$ & 0,44 \\
\hline & & Densidade $\left(\mathrm{kg} / \mathrm{mm}^{3}\right)$ & 7800 \\
\hline
\end{tabular}

O modelo da estrutura foi analisado utilizando o software comercial ANSYS. A viga foi modelada utilizando o elemento SOLID45 e discretizada em 105 elementos sólidos de 8 nós, com graus de liberdade de deslocamento nas direções $\mathrm{x}, \mathrm{y}$ e $\mathrm{z}$, e para $\mathrm{o}$ PZT foi utilizado o elemento SOLID5, discretizado em 100 elementos sólidos de 8 nós com graus de liberdade nas direções $\mathrm{x}, \mathrm{y}$ e $\mathrm{z}$ e potencial elétrico. Através de análise modal teórica, obteve-se a frequência natural do primeiro modo igual a $30,666 \mathrm{~Hz}$. $\mathrm{O}$ potencial elétrico gerado pelo modelo foi calculado através de simulações numéricas, considerando o amortecimento proporcional $\left(\alpha=0,7\right.$ e $\left.\beta=6 \times 10^{-4}\right)$ e uma excitação pela base. Foi utilizada uma aceleração senoidal de amplitude $9,81 \mathrm{~m} / \mathrm{s}^{2}$ e o potencial elétrico gerado foi de $11,9 \mathrm{~V}$ pico a pico. Definido o primeiro conversor passou-se a segunda etapa referente à definição da estrutura multifrequência.

\subsubsection{Definição da faixa de frequências}

A faixa de frequências de operação da piezoestrutura multifrequência foi definida de forma a absorver as oscilações em torno da frequência natural do primeiro modo de vibração da piezoestrutura de $30,6 \mathrm{~Hz}$. Neste caso, foi utilizado o conceito de banda de meia potência para estimar a faixa de frequências que a piezoestrutura pudesse operar produzindo uma razoável quantidade de energia. Essa faixa de frequências foi definida como a banda de frequência de meia potencia da curva de ressonância, isto é, a frequência em que a amplitude da curva de ressonância nas proximidades do pico tenha um valor equivalente ao $\left(V_{\text {max }} / \sqrt{2}\right)$, apresentando uma redução de $3 \mathrm{~dB}$.

$$
d B=20 * \log _{10}\left(\frac{V}{V_{\text {ref }}}\right)
$$

Onde Vref é a tensão elétrica produzida na ressonância, ou seja em $30,6 \mathrm{~Hz}$.

Uma varredura em frequências foi realizada e estimadas as respectivas tensões geradas para cada condição, Tabela 2.

Tabela 2. Dimensões e propriedades do conversor piezelétrico.

\begin{tabular}{|l|c|c|c|c|c|c|c|}
\hline Freq. (Hz) & 27 & 28 & 29 & 30 & 31 & 32 & 33 \\
\hline Tensão (V) & 7.84 & 8.48 & 10.2 & 11.5 & 10.3 & 8.64 & 7.6 \\
\hline
\end{tabular}

Com os valores obtidos na Tabela 2, utilizou-se a Equação 5 para determinar a faixa de frequências entre $27 \mathrm{~Hz}$ e $33 \mathrm{~Hz}$ correspondente a $68,17 \%$ e $66,08 \%$, respectivamente da tensão elétrica gerada na ressonância, conforme Figura 2.

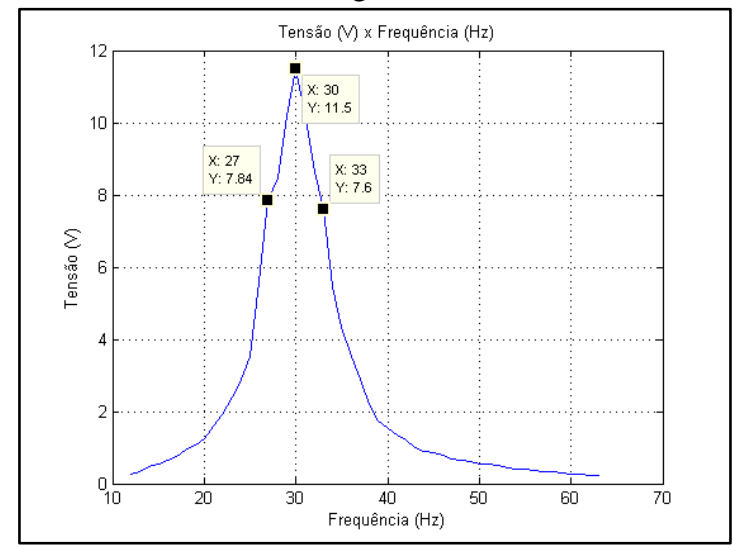

Figura 2. Varredura das tensões elétricas geradas em cada frequência.

\section{Comparação dos modelos}

Para validar o modelo numérico foi realizado um teste experimental onde o conversor piezelétrico foi submetido às mesmas condições utilizadas nas simulações buscando avaliar a correlação entre os modelos. Na Figura 3 é apresentado o aparato experimental utilizado nos testes. Nos testes experimentais uma onda senoidal de $30,01 \mathrm{~Hz}$ foi gerada no gerador de sinais sendo sua amplitude amplificada até uma amplitude equivalente à $9,81 \mathrm{~m} / \mathrm{s}^{2}$ através do amplificador de sinais. O sinal é transmitido ao shaker eletrodinamico que excita a estrutura piezelétrica através de sua base. Foi utilizado um acelerômetro para monitorar a amplitude de vibração.

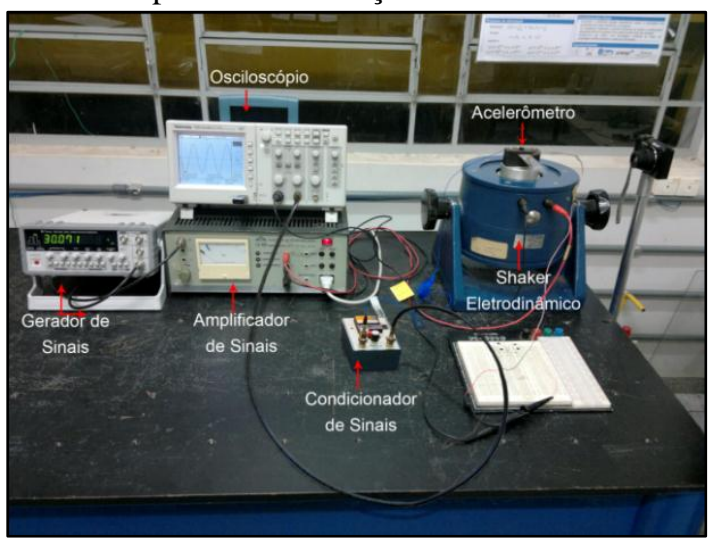

Figura 3. Aparato experimental de validação dos resultados numéricos.

A Tabela 3 mostra os valores numérico e experimental da tensão gerada quando a estrutura foi excitada numa condição de ressonância. Neste caso, a correlação dos modelos se mostra bastante adequada.

Tabela 3. Dimensões e propriedades do conversor piezelétrico.

\begin{tabular}{c|c|c|c}
\hline Método & Numérico & Experimental & Diferença (\%) \\
\hline Freq. $f_{\mathrm{n}}(\mathrm{Hz})$ & 30,666 & 30,01 & 2,18 \\
Tensão $(\mathrm{V})$ & 11,9 & 11,5 & 3,47 \\
\hline
\end{tabular}




\section{Estudo Paramétrico das Variáveis Geométricas}

Neste tópico foram realizadas varias simulações variando-se a espessura, largura e comprimento da viga hospedeira de modo a determinar quais características geométricas da estrutura piezelétricas são mais apropriadas para alterar a frequência natural do conversor piezelétrico mantendo um tamanho compacto. Pelas simulações observou-se que variando-se a espessura de acordo com valores comerciais das placas de acrílico $(1.1 \mathrm{~mm}, 2 \mathrm{~mm}$ e $3 \mathrm{~mm})$ as frequências naturais das vigas hospedeiras ficam fora da faixa pré-determinada. Variando-se a largura (18mm, $20 \mathrm{~mm}$ e $22 \mathrm{~mm}$ ) foi possível observar que não há muita influência na variação das frequências naturais. Entretanto, quando se varia o comprimento (102.5mm, $107.5 \mathrm{~mm}$ e $112.5 \mathrm{~mm})$ foi possível observar que as frequências naturais de cada conversor piezelétrico da piezoestrutura multifrequência estão dentro da faixa determinada.

Como um dos objetivos deste trabalho é também gerar o máximo de energia elétrica possível, novas simulações foram realizadas onde variou-se o comprimento do elemento piezelétrico de modo a verificar em qual comprimento se obtém maior energia. $\mathrm{Na}$ Figura 4 são apresentadas as tensões elétricas de pico geradas em função do comprimento do elemento piezelétrico.

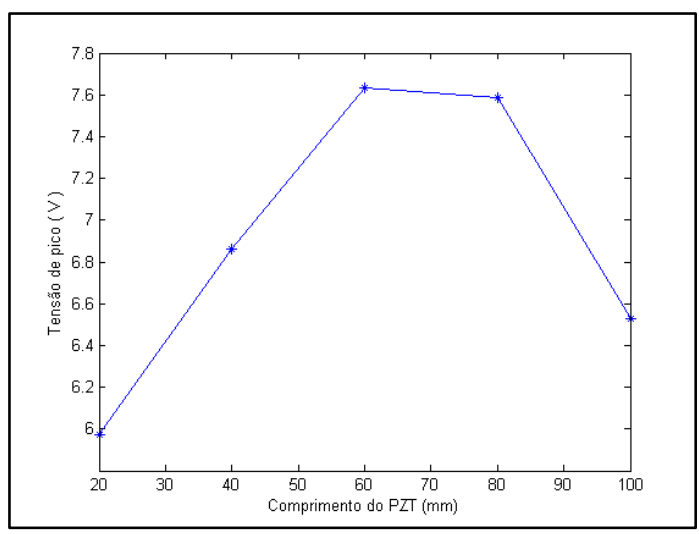

Figura 4. Tensão de pico em função do comprimento do PZT.

É possível observar que a maior energia gerada ocorre no elemento piezelétrico com comprimento igual a $60 \mathrm{~mm}$. Todo o procedimento foi realizado novamente de modo a identificar e determinar a configuração final da piezoestrutura multifrequência com um elemento piezelétrico de $60 \mathrm{~mm}$ acoplado em cada uma das vigas hospedeiras da estrutura piezelétrica. Buscando avaliar a potência elétrica disponível em cada um dos conversores piezelétricos da estrutura multifrequencia novas simulações foram realizadas onde uma resistência elétrica foi conectada nos terminais do elemento piezelétrico. Os valores da resistência foram variados entre $1 \mathrm{~K} \Omega$ e $1 \mathrm{M} \Omega$ utilizando-se apenas de valores comerciais de resistência. Para o cálculo da potência disponível no elemento piezelétrico foi considerada a seguinte equação:
(6)

Na Figura 5 é apresentada a potência disponível em cada um dos conversores piezelétricos da piezoestrutura multifrequência com elemento piezelétrico de $60 \mathrm{~mm}$ de comprimento.

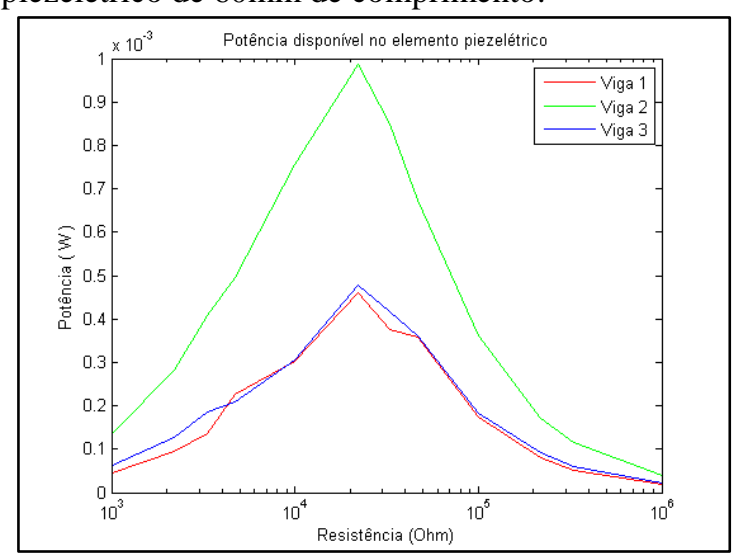

Figura 5. Potência disponível no elemento piezelétrico de cada uma das vigas.

\section{Conclusão}

Após todas as simulações, a análise dos resultados revela que enquanto a piezoestrutura multifrequência for excitada na frequência de $30 \mathrm{~Hz}$ um dos conversores estará na ressonância e os outros dois conversores gerando energia $60 \%-70 \%$ do valor do outro conversor na ressonância. Caso a frequência de excitação oscile, se distanciando da frequência de ressonância, os conversores piezelétricos adicionais mantém o nível de energia gerada em um nível aceitável. A técnica aplicada e configuração proposta se mostram como uma solução atrativa para tentar minimizar as perdas na geração de energia em ocasiões de variação na frequência de excitação.

\section{Agradecimentos}

Os autores agradecem ao CNPq e à FAPEMIG pelo financiamento parcial do presente trabalho de pesquisa através do INCT-EIE.

\section{Referências Bibliográficas}

Adachi, K.; Tanaka, T. An experimental power generation evaluation of cantilever type of piezoelectric vibration energy harvester. SMASIS, 2009, Oxnard. Proceedings... Oxnard: ASME, 2009. p. 281-289.

Ajitsaria, J.; Choe, S. Y.; Shen, D.; Kim, D. J. Modeling and analysis of a bimorph piezoelectric cantilever beam for voltage generation. Smart Materials and Structure, Bristol, v. 16, n. 2, p. 447-454, 2007. DOI: 10.1088/0964-1726/16/2/024

Dutoit, N. E.; Wardle, B. L. Experimental verification of models for microfabricated piezoelectric 
vibration energy harvesters. AIAA Journal, Newport, v. 45, n. 5, p. 1126-1137, 2007. DOI: $10.2514 / 1.25047$

Erturk, A.; Inman, D. J. Issues in mathematical modeling of piezoelectric energy harvesters. Smart Materials and Structures, Bristol, v. 17, n. 6, p. 14, 2008b.

Ferrari, M.; Ferrari, V.; Guizzetti, M.; Marioli, D. An autonomous battery-less sensor module powered by piezoelectric energy harvesting with RF transmission of multiple measurement signals. Smart Materials and Structures, Bristol, v.18, n. 8, 9 p., 2009.

Johnson, D. E. Design of an ultra-low power control system for a self-powered wireless sensor. 2006. Dissertação (Mestrado) - University of Florida, Florida, 2006.

Kok, S. L.; Mohamad, N.; Weng, D. Y. F.; Kien, C. $\mathrm{S}$;; Fu, D. C. Multifrequency energy harvesting using thick-film piezoelectric cantilever. In: International Conference on Electrical, Control and Computer Engineering, 2011, Malaysia. Proceedings... Malaysia: INECCE, 2011.

Lefeuvre, E.; Badel, A.; Richard, C.; Petit, L.; Guyomar, D. A comparison between several vibration powered piezoelectric generators for standalone systems. Sensors and Actuators A, Lyon, v. 126, p. 405-416, 2006.

Ramadass, Y. K.; Chandrakasan, A. P. An efficient piezoelectric energy harvesting interface circuit using a bias-flip rectifier and shared inductor. IEEE Journal of Solid-State Circuits, Piscataway, v. 45, n. 1, p. 189-204, 2010.

Souza, F. S. Sistema de extração de potência (power harvesting) usando transdutores piezelétricos. 2011. Dissertação (Mestrado) - Faculdade de Engenharia, Universidade Estadual Paulista, Ilha Solteira, 2011. 